\title{
Sensitivity Enhancement of Bimaterial MOEMS Thermal Imaging Sensor Array using 2- $\lambda$ readout
}

\author{
O. Ferhanoğlu, H. Urey \\ Koç University, Electrical Engineering, Istanbul-TURKEY
}

\begin{abstract}
Diffraction gratings integrated with MEMS sensors offer sensitive displacement measurements. However, the sensitivity of the interferometric readout may drop significantly based on the sensor position. A two wavelength readout method was developed and tested previously in order to maintain the sensitivity of the readout $>\% 50$ maximum sensitivity over a broad range (i.e. several um's for visible wavelengths). This work demonstrates the sensitivity enhancement of a MEMS thermal imaging sensor array. Measurement of the target scene was performed using two lasers at different wavelengths $(633,650 \mathrm{~nm})$. The diffracted $1^{\text {st }}$ order light from the array was imaged onto a single CCD camera for both sources. The target scene was reconstructed by observing the change in the $1^{\text {st }}$ diffracted order diffraction intensity for both wavelengths. Merging of the data, acquired with two different sources, is performed by assigning each pixel in the final image with the higher sensitivity pixel among two measurements. $>30 \%$ increase in the average sensitivity was demonstrated for the sensor array.
\end{abstract}

Keywords: Grating Interferometry, Diffraction orders, Two-wavelength interferometry, MEMS, Thermal imaging sensor array, CCD camera.

\section{INTRODUCTION}

Diffracting gratings embedded with MEMS sensors provide sub-nm displacement measurements for various sensing and imaging applications [1-5]. Despite their ultra-high sensitivity, interferometer based readouts are limited to an unambiguous range of $\lambda / 4$ for a single, narrowband illumination source. Integrating multiple sources into the readout, enhances both the range and the sensitivity of the measurement. Extended range performance on a single lamellar grating Fourier Transform Infrared Spectrometer was previously demonstrated using two sources [6]. This work highlights another aspect of two wavelength interferometry which is the enhancement of sensitivity for an array of sensors.

\section{GRATING INTERFEROMETRY}

Fig. 1(a) illustrates a diffracting grating based sensor. The sensor consists of an embedded fixed grating, placed underneath, and a reflector that is placed on top of the sensor. The illumination is performed from the bottom side. Fixed grating and the reflector, forms an interferometer where the movement of the sensor causes modulation of the optical path difference between the grating and the reflector, which could be monitored with a photo detector. Well known formulas for the $0^{\text {th }}$ and odd diffracted order light can be expressed as:

$$
\begin{aligned}
& I_{0} \alpha\left(\cos \left(\frac{2 \pi g}{\lambda}\right)\right)^{2} \\
& I_{\text {odd }} \alpha\left(\sin \left(\frac{2 \pi g}{\lambda}\right)\right)^{2}
\end{aligned}
$$

Where $g$ is the gap between the sensor and the fixed grating and $\lambda$ is the wavelength of illumination. Since the intensity variation of all orders is periodic with respect to gap, the range of an interferometric readout is bounded to $\lambda / 4$. Furthermore, the sensitivity, slope of the intensity vs. gap behavior, can severely diminish at peaks and dips of the sinusoidal intensity curve. This work demonstrates a $2-\lambda$ readout method to improve the sensitivity for an array of sensors.

\footnotetext{
Optical Micro- and Nanometrology III, edited by Christophe Gorecki, Anand Krishna Asundi, Wolfgang Osten,
} Proc. of SPIE Vol. 7718,771800 - ( 2010 SPIE · CCC code: 0277-786X/10/\$18 · doi: 10.1117/12.854753 
Fig 1. (b) illustrates the concept of two wavelength readout method of a sensor array, where the diffracted orders $\left(1^{\text {st }}\right.$ diffracted order for the figure) are captured by a CCD camera. In the setup, the sensor array is illuminated by two lasers with wavelengths $\lambda_{1}$ and $\lambda_{2}$, with time multiplexing. In other words the sensor array is illuminated by $\lambda_{1}$ at even frames, and $\lambda_{2}$ at odd frames. For every sensor, the illumination will result in different intensity level $(I)$ and the sensitivity at a certain gap $(\mathrm{g})$, sensitivity is defined as. $\mathrm{S}(\lambda, \mathrm{g})=\mathrm{d} / / \mathrm{dg}$. Overall sensitivity of the proposed scheme is :

$$
S_{2 \lambda}\left(\lambda_{1}, \lambda_{2}, g\right)=\max \left\{S\left(\lambda_{1}, g\right), S\left(\lambda_{2}, g\right)\right\}
$$

Where $S_{2 \lambda}$ is the sensitivity of a sensor under two wave illumination and takes on the higher sensitivity value of the two sensitivity measurements performed using $\lambda_{1}$ and $\lambda_{2}$ separately. A calibration routine is necessary before the measurement to identify the sensitivity achieved for each sensor in the array and for each wavelength. At the measurement stage, the sensor array is illuminated by both wavelengths one at a time, however only the higher sensitivity measurement is used for each sensor.

Selection of $\lambda_{1}$ and $\lambda_{2}$ plays critical role on the amount of measurement range and sweet spot of sensitivity. The measurement range is proportional with the smallest common multiple of the two wavelengths and the the highest overall sensitivity $\left(\mathrm{S}_{2 \lambda}\right)$ is achieved in the middle of the range [6].

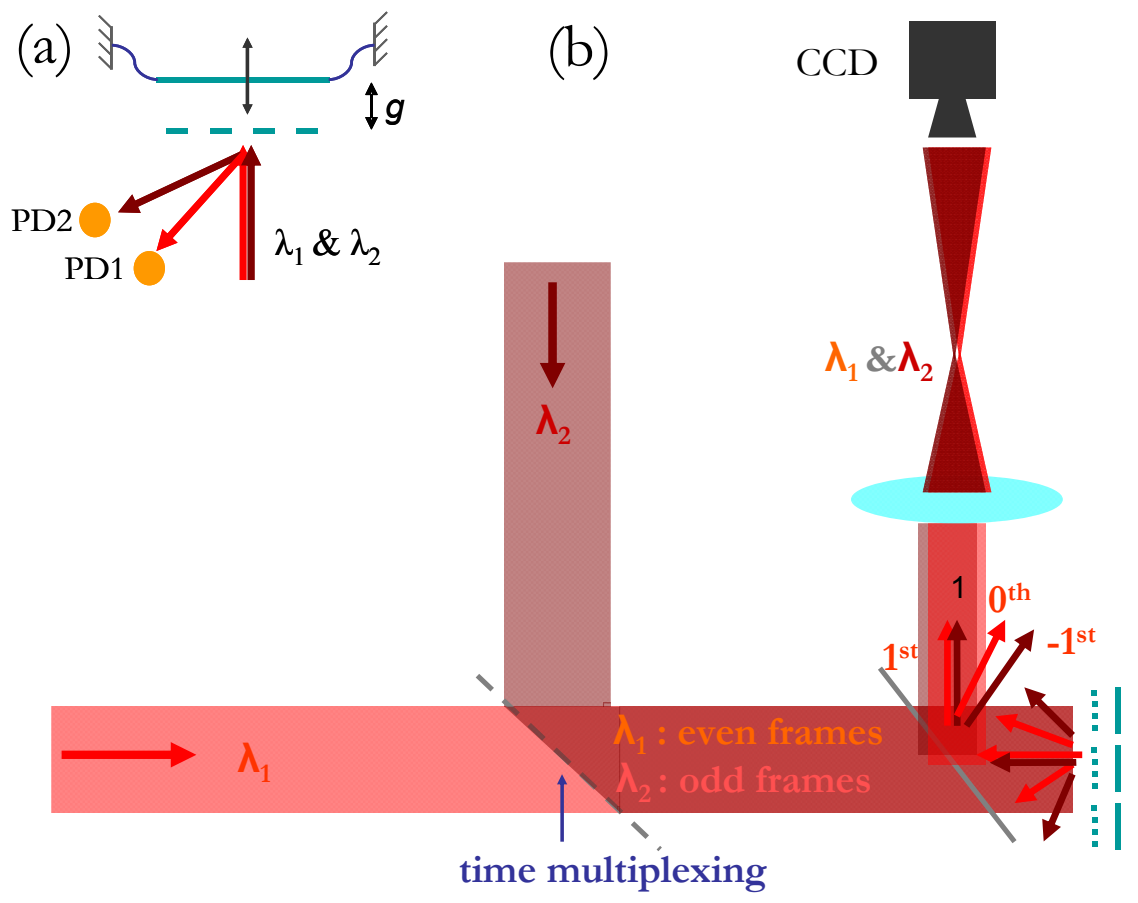

Fig 1. (a) Diffraction grating based MEMS Sensor that is illuminated with two lasers with wavelengths $\lambda_{1}$ and $\lambda_{2}$ (b) Proposed method using two - wavelength illumination where the sensor array is illuminated by two sources, one at a time. The diffracted $1^{\text {st }}$ order light is imaged onto an array of photodetectors, i.e a CCD camera.

\section{EXPERIMENTAL SETUP AND RESULTS}

\subsection{Thermal Imaging Sensor Arrays}

Sensitivity enhancement was demonstrated using previously fabricated thermal imaging sensor arrays [7]. Fig. 2 (a) illustrates cartoon drawing of a thermal imaging sensor. The sensor, having 50x50 um size, is composed of an Infrared (IR) absorbing membrane (200 $\mathrm{nm} \mathrm{SiNx})$, bimaterial legs $(100 \mathrm{~nm}$ aluminum) that bend due thermal mismatch of its 
layers with temperature change and a diffraction grating that is fabricated underneath. The sensor bends in response to the target temperature, which is monitored by observing the $1^{\text {st }}$ diffracted order with a photodetector or a CCD camera for an array of sensors. Fig. 2 (b) and (c) illustrate the finite element model and microscope images of the sensor(s).

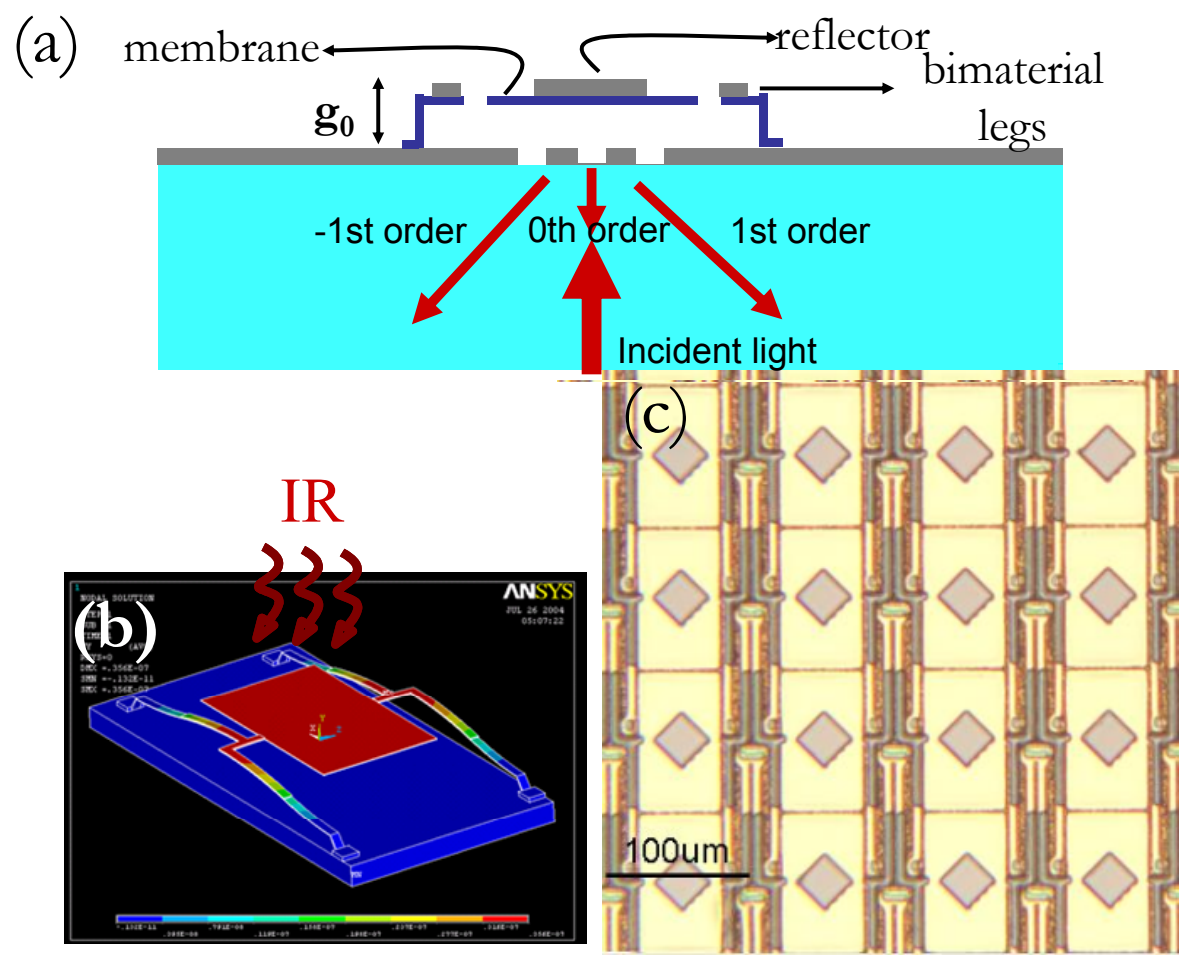

Fig 2. (a) Diffraction grating based Thermal Imaging Sensor (side view) (b) SEM image (c) microcope image of an array of sensors

Fabrication of the sensor array was performed by the Microelectronics Center (METU-MET) at the Middle East Technical University- Ankara, Turkey. Fig 3. illustrates the fabrication steps, which were also described previously in [7]. The process starts with deposition and patterning of $\mathrm{Ti} / \mathrm{Au}$ gratings on pyrex substrate. Polyimide is spinned and patterned as a sacrificial layer. $200 \mathrm{~nm} \mathrm{SiN}_{\mathrm{x}}$ is deposited to serve as both the structural and IR absorbing layer. $300 \mathrm{~nm}$ Aluminum is deposited on the legs as a thermal mismatch layer. A thin layer of titanium nitride (TiN) is deposited after patterning of aluminum layer, to enhance IR absorption. Fig. 3, summarizes the details of fabrication steps of the thermal imaging sensor arrays. 


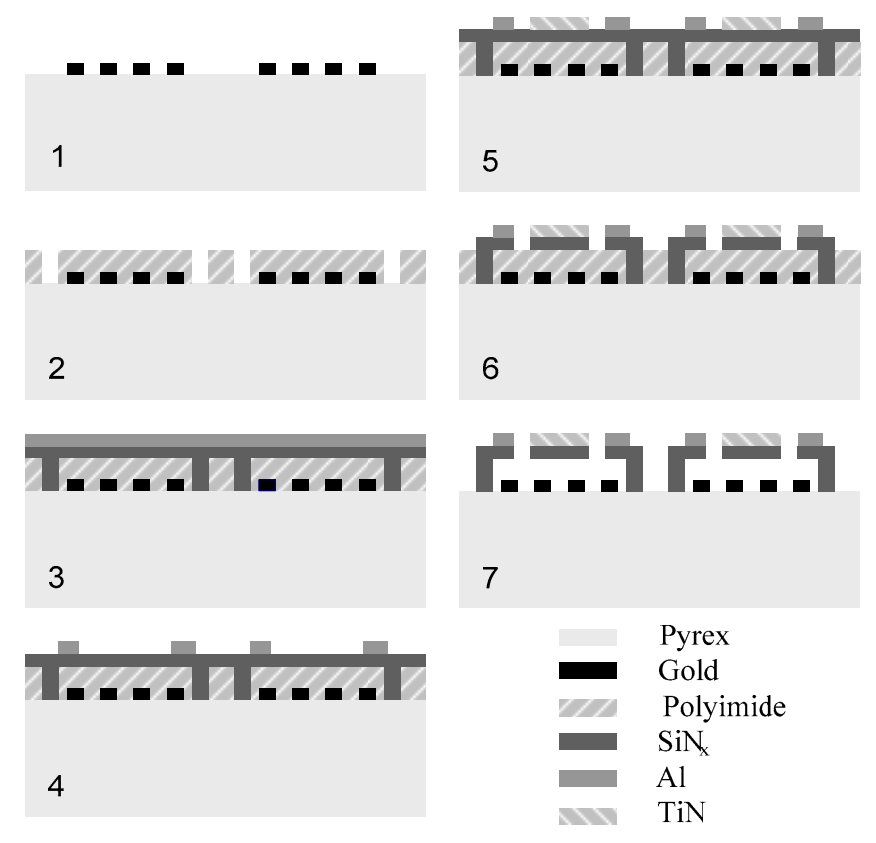

Fig. 3: Fabrication steps [7]:

1) Patterning of gold gratings

2) Polyimide spinning and definition of anchors

3) $\mathrm{SiN}_{\mathrm{x}}$ and $\mathrm{Al}$ deposition

4) Al patterning

5) TiN deposition and patterning

6) $\mathrm{SiN}_{\mathrm{x}}$ patterning

7) Release

\subsection{Experimental Results}

A 160x120 array of thermal imaging sensors were illuminated by two laser sources, of wavelengths 633 and $650 \mathrm{~nm}$ in the configuration shown in Fig. 1 (b). The sensor array required vacuum operation. Furthermore an IR lens was placed at the front side of the sensor array to image thermal information of the target. A periodic slab PCB, illustrated in Fig. 4 (a) was placed in front of an IR heater. The IR heater was effectively turned OFF and ON by placing a metal plate in front of the lens. Images were acquired by both sources while IR heater was ON and OFF. The difference images were calculated for both wavelengths, which are illustrated in Fig. 4 (b) and (c). The sensitivity histograms shown in Fig. 4 (e) and (f) are displayed based on the areas shown with white rectangles, which correspond to the openings in the periodic slab. Finally the combined image (Fig. 4 (d)) and histogram (Fig 4. (g)) are calculated based on "winner takes all" algorithm that is depicted in Eq. 3. A clear enhancement of over 30\% is observed in the average sensitivity of the histogram. 


$$
\begin{aligned}
& \lambda 1=650 \mathrm{~nm} \\
& \lambda 2=633 \mathrm{~nm}
\end{aligned}
$$

$\lambda 1$
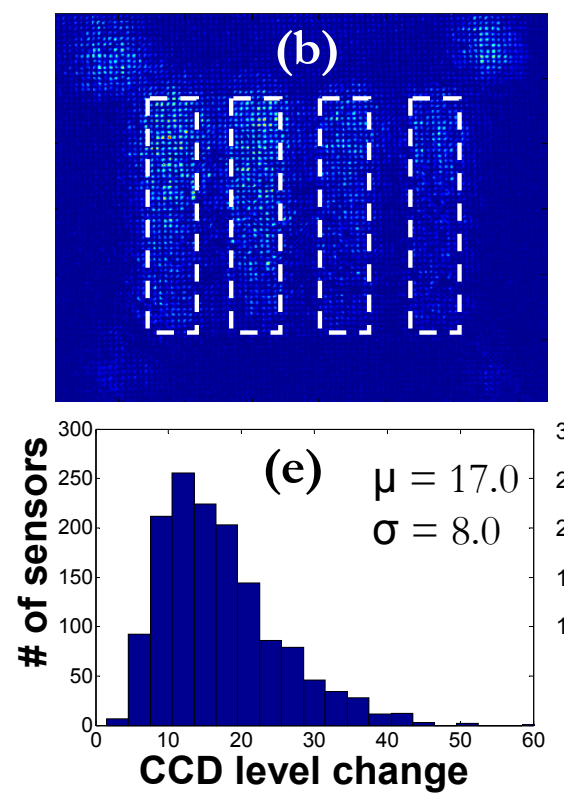

\section{(a)}
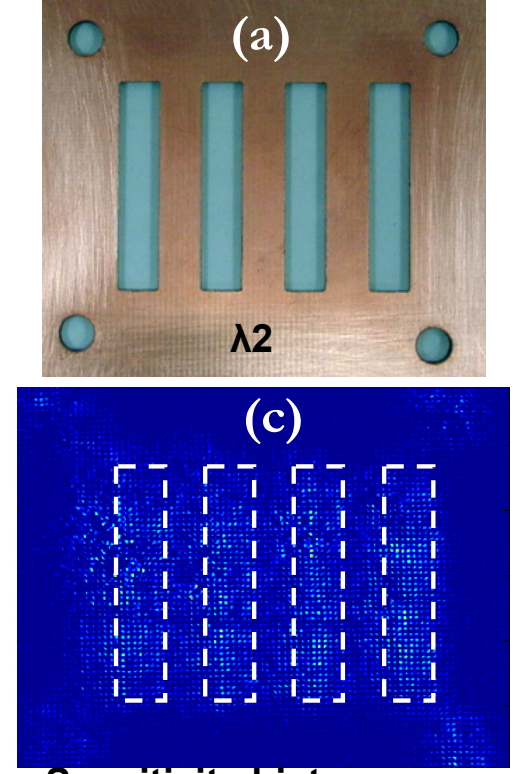

${ }_{300}$ Sensitivity histograms

(f) $\mu=17.0$ $\sigma=7.2$

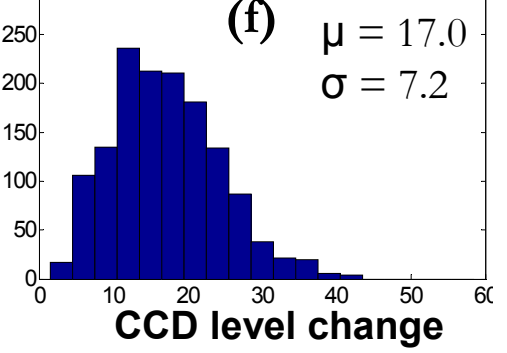

$\lambda 1 \& \lambda 2$ combined
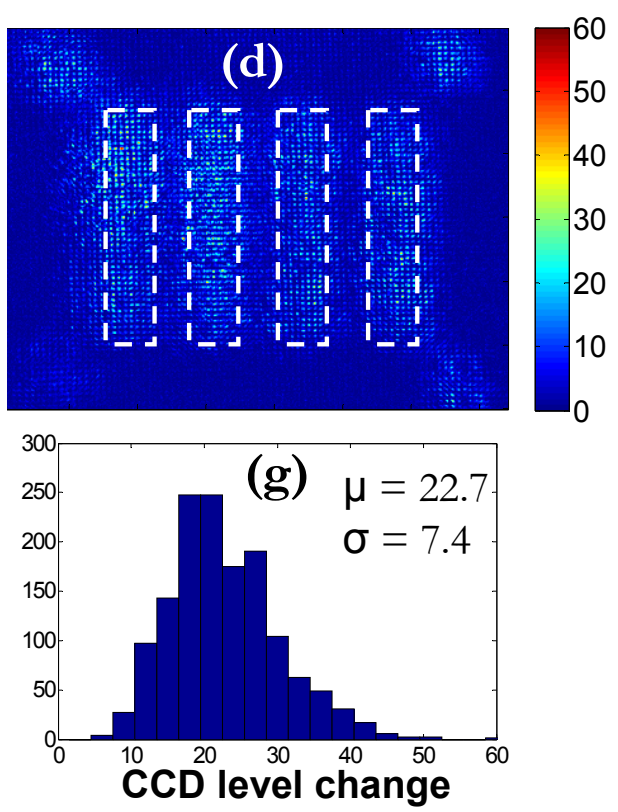

Fig 4. (a) Target used in the experiment: periodic PCB slab, that is placed in front of an IR heater. (b) Difference image acquired by $\lambda_{1}$ (c) Difference image acquired by $\lambda_{2}$ (d) combined image (e) Histogram of $\lambda_{1}$ difference image (f) Histogram of $\lambda_{2}$ difference image (d) Histogram of combined image. The average sensitivity increases from 17 CCD levels for each individual image up to 22.7 CCD levels. 


\section{CONCLUSIONS AND DISCUSSION}

A two wavelength measurement was performed on a thermal imaging sensor array. $>30 \%$ increase in average sensitivity is demonstrated and validated by theory. Real time measurement with the proposed method is to be realized by imaging diffracted order(s) of the two sources onto the same CCD / CMOS camera and taking data of one source at a time. This time multiplexing scheme may be implemented using various methods such as synchronous modulation and capture of both sources or by placing a rotating optical filter before CCD plane. Imaging two sets of diffracted order light on the same are of the CCD, requires critical alignment of the beam splitter, lenses and fourier filtering. Besides thermal imaging sensor arrays, this method can be adapted to a variety of sensing application such as biosensor arrays, grating spectrometers and atomic force microscopy for improving measurement range and sensitivity

\section{REFERENCES}

[1] G.G. Yaralioglu, S .R. Manalis, A. Atalar, C.F. Quate, "Analysis and design of an interdigital cantilever as a displacement sensor," J. Appl.Phys.,83, pp. 7405-7415 (1998).

[2] A.G. Onaran, M. Balantekin, W. Lee, W.L. Hughes, B.A. Buchine, R.O. Guldiken, Z. Parlak, C.F. Quate and F.L. Degertekin, "A new atomic force microscope probe with force sensing integrated readout and active tip," Review of Scientific Instruments, 77, 023501, 2006

[3] C. Ataman, H. Urey, A. Wolter, "MEMS-based Fourier Transform Spectrometers" J. Micromechanics and Microengineering, Vol 16, pg 2516-2523, 2006

[4] H Torun, J Sutanto, K K Sarangapani, P Joseph, F L Degertekin, C Zhu, "Micromachined membrane-based active probe for biomolecular mechanics measurement ", Nanotechnology, 18 (2007) 165303C. Jones, Director, Miscellaneous Optics Corporation, interview, Sept. 23, 2008.

[5] A. Ozturk et al, "A magnetically ${ }^{7}$ A. Ozturk et al, "A magnetically actuated resonant mass sensor with integrated optical readout", Photonics Technology Letters Vol. 20, 1905-1907, 2008

[6] O. Ferhanoglu, M. F. Toy, H. Urey, "Two-wavelength Grating Interferometry for MEMS Sensors," IEEE Photonics Tech. Lett., Vol. 19, p. 1895-1897, 2007

[7] M. F. Toy, O. Ferhanoglu, H. Torun, H. Urey, "Uncooled Infrared Thermomechanical Detector Array: Design, Fabrication, and Testing," Sensors and Actuators: A. Physical, doi:10.1016/j.sna.2009.02.010 2009 